\title{
Vibration Attenuation and Joint Navigation through Robust Control System Hardware for Multi-Degrees-of-Freedom Flexible Robots
}

\author{
Pratik Chothe $^{1 *}$, Shreyash Gajlekar ${ }^{2}$, Aniket Bhelsaikar ${ }^{1}$, Viinod Atpadkar ${ }^{1}$, Nilesh Uke ${ }^{3}$ and Debanik Roy \\ ${ }^{1}$ SVR Infotech, Pune, India \\ ${ }^{2}$ Tata Technologies, Pune, India \\ ${ }^{3}$ Trinity Academy of Engineering, Pune, India \\ ${ }^{4}$ Division of Remote Handling \& Robotics, Bhabha Atomic Research Centre \& Homi Bhabha National Institute, Department of Atomic \\ Energy, Mumbai, India \\ *Corresponding Author: Pratik Chothe, SVR Infotech, Pune, India.
}

Received: December 27, 2021; Published: January 12, 2022

\begin{abstract}
The inherent vibration occurring due to compliance of Flexible Robotic System (FRS) provides numerous challenges to achieve accuracy at the end-effector of the FRS. This paper focuses on the study of influence of electronic control system hardware over electromechanical output of the FRS. The paper provides an insight to the relationship between drive current and motor torque with vibrations in the system. Design details of the novel indigenously-developed control electronics and, finally the controller of the prototype FRS will be reported in this paper, backed up by the glimpses of mechanical hardware. The performance and robustness of the prototype FRS will be finally discussed based on some representative analysis and results thereof. We will discuss about implementing various trajectory functions in the control program of the prototype FRS. The paper will further lead to modified linear trajectory where parabolic blends are introduced to reduce the infinite acceleration, which would eventually reduce the vibration in the links of the FRS.
\end{abstract}

Keywords: Flexible Robot; Control System; Electronics; Vibration; Rheology; Sensor; Gripper; Trajectory Functions

\section{Introduction}

The prevalence of in-situ vibration of Flexible Robotic System (FRS), in the form of trembling of its links and/or twisting of the joints, pose critical bottlenecks in real-time control of the FRS so far as attainment of accuracy of end-of-arm tooling is concerned. Often, we find this in-situ trembling, especially in case of multi-degrees-of-freedom FRS, as prolonged jitter. Hence, we need to go for suitable vibration harnessing mechanism, ideally through the system electronics before deploying such FRS in application-field. The vibration reduction paradigm of FRS uses two distinct approaches of control system, namely: a] input command shaping and b] closedloop feedback for vibration control. In past, researchers have examined the closed-loop feedback system for reduction of vibration at the end-effector of FRS. In order to reduce the vibration of the prototype FRS, having three slender links with three revolute joints inter-spaced, the first method of input command shaping has been used. Input command shaping involves chain of commands that do nullify its own vibrations. And, to achieve this, our work focuses on velocity and torque shaping of the flexible links of the prototype FRS. Vibration in the prototype FRS is self-propagating in nature and does not follow analytical modelling \&rule-base in real-time applications. The prototype FRS is a three degrees-of-freedom (d.o.f.) robotic system involving three revolute joints, three links, two flexible shafts, a recirculating ball screw, a sensor-instrumented miniature gripper, a tripod and electrical actuators, motion controllers \& drivers. In order to implement input command shaping on the prototype FRS it is essential to know the nature of the built-in vibration. Finite Element Analysis (FEA) was exercised in order to find vibrational characteristics of the revolute joints of the prototype FRS.

Citation: Pratik Chothe., et al. "Vibration Attenuation and Joint Navigation through Robust Control System Hardware for Multi-Degrees-of-Freedom Flexible Robots". Medicon Engineering Themes 2.2 (2022): 03-17. 
The design paradigm of a typical multi-degrees-of-freedom FRS is essentially task-driven, wherein one end of the manipulator is connected to the supporting base while the other end is attached to the end-effector. The relative motions of the joints give the position of the FRS-links in desired orientations. The kinematics of the flexible robot arm deals with the motion of the robot with respect to the fixed reference co-ordinate system. As a matter of fact, it is quite interesting to know a-priori whether there are any obstacles present in the path, prescribed for the FRS.

The control problem of a flexible manipulator is typical divided into two coherent sub-problems, namely: the trajectory planning and the motion control. Nonetheless, real-time control of inherent randomized vibration of a prototype FRS becomes a challenging problem indeed, if it has multiple degrees-of-freedom. The novelty of our control system architecture is rooted through joint navigation paradigm using trajectory-based control in real-time.

Major research efforts have been put forward, over the past few decades to model and control flexible robotic systems with varying degrees-of-freedom. Various research modules, dealing with novel control systems for FRS have been documented in recent past. Singer \& Seering [1] demonstrated the preshaping of input for reduction of vibration in FRS. A new approach for dynamic modelling of multi- d.o.f. FRS was reported in [2], while the same was tackled through spring and rigid bodies in [3]. The issues of frequency damping and control of vibration in a single-link FRS thereof were studied through adaptive resonant control of the variables [4], which was further strengthened by using novel methods for algebraic identification [5] and integral resonant control [6]. Smith [7] proposed input shaping as technique for generating non-oscillatory response from a lightly-damped system subject to a step input. The response is being achieved by generating two transient oscillations such that the end-result is cancellation of oscillations. In its earliest form, command preshaping was used as motion templates for flexible manipulators as well as perturbation techniques [8]. Junkins \& James [9] and Chun et al [10] presented practical solutions of the optimal control formulation for flexible systems. Gupta [11] presented frequency shaping terms in the optimal formulation of the feedback control equation of FRS, which was substantiated by the closed-form solution approach by Juang et al [12]. Artificial intelligence model, using fuzzy logic for control of FRS, was proposed by Moudgal et al [13]. A treatise on various available techniques for the control of FRS can be referred for new research\& application-based work [14].

It may be commented that while real-time control of multi-d.o.f. is a real challenge for the researchers, a pretty large number of papers on the control semantics of single-link FRS (with fixed and variable payload at end-effector) have been published till date. Kotnik et al [15] presented the acceleration feedback control for a single-link FRS, based on experimental results. A considerable number of researches have been conducted on the modelling and control of 2 d.o.f. flexible systems as well [16-19]. In comparison to the research conducted with single and two-link FRS, 3-link FRS has its own set of challenges. Feliu et al [20] explained the modelling of 3-d.o.f. planar FRS with feedback control and inverse kinematics in spherical co-ordinates. Somolinos et al [21] reported how a FRS-arm can be modelled as a structure with only one oscillation mode in each of the three orthogonal directions. Indigenous design, analysis and successful hardware development of a test-bed prototype of a novel 3-d.o.f. FRS, fitted with a mini-gripper,have been reported by our group [22-24].

Vibrational analysis becomes a critical requirement for real-time control of multi-d.o.f. FRS because of its slender length and incorporation of flexible shafts. As a matter of fact, the transmission of motor-power in FRS-links is a distinct source of vibration. While the system is in motion, the vibration gets induced in the assembly that gets further intensified by the augmentation of flexible shafts. The ensemble cantilever structure of the planar FRS dictates the amount of such in-situ vibration as well as its modal frequencies. Thus, attenuation of vibration requires very robust control system \& program level manipulations for vibration reduction and achieving the required precision at the end-effector.

The paper has been organized in six sections. Ab initio vibrational analysis of the prototype FRS, unfolding its nature \& characteristics, is reported in the next section. The schematic and syntax of the control system architecture and design of device controller of the prototype FRS are attributed in section 3. Section 4 addresses the real-time programming semantics of the prototype FRS, along with the Graphical User Interface (GUI) developed. An overview of the hardware architecture of the controller of the prototype FRS is provided in section 5 and finally section 6 concludes the paper.

Citation: Pratik Chothe., et al. "Vibration Attenuation and Joint Navigation through Robust Control System Hardware for Multi-Degrees-of-Freedom Flexible Robots". Medicon Engineering Themes 2.2 (2022): 03-17. 


\section{Vibration Analysis of the Prototype Flexible Robotic System}

The prototype three d.o.f. planar serial-chain FRS, with a horizontal span of $1500 \mathrm{~mm}$, is the source of in-situ vibration as well as trembling / jitter due to its jointed disposition \& slenderness of the links. Hence, by virtue of the self-weight itself the FRS will have mild shaking that will be a precursor of natural means of generating vibration in real-time. This self-vibrating structure will be boosted up for enhanced vibration as and when the prototype FRS will grip a payload by its mini-gripper. We have modeled the prototype system for a maximum payload of $800 \mathrm{gm}$ at a terminal reach of $1500 \mathrm{~mm}$ and analyzed the same through finite element method. The total weight of the prototype FRS assembly is $18 \mathrm{~kg}$ (approx.), considering a factor of safety of 2.0 for strength-bearing members.

FE-simulation was carried out on each one of the three links of the prototype FRS upto 10 modes of vibration, considering base as fixed. All those modal frequencies (in Hz.) are plotted in fig. 1 and indexed. These data are useful for extracting the overall dynamic behaviour of the FRS at a certain frequency.

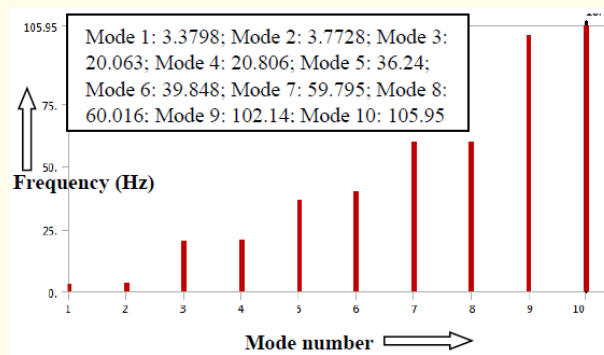

Figure 1: Plot of Modal Frequencies under Vibration of the FRS.

Figure 2 shows the deformation pattern of the prototype FRS under first mode of vibration (modal frequency of 3.3798Hz).

The ensemble deformation pattern of fig. 2, in the form of non-linear deflection, indicates that the effect of payload is more prudent at the third link zone but it is considerable at the second link too. Although natural frequency of vibration of the system at the base mode is controllable, it needs to be compared with the other modal frequencies as well.

The deformation pattern of the FRS will be altered to some extent under next higher modes of vibration, i.e., $2^{\text {nd }} \& 3^{\text {rd }}$ modes. While maximum deformation values get reduced, the effect of deformation is more spreading over the links during these modes of vibration.

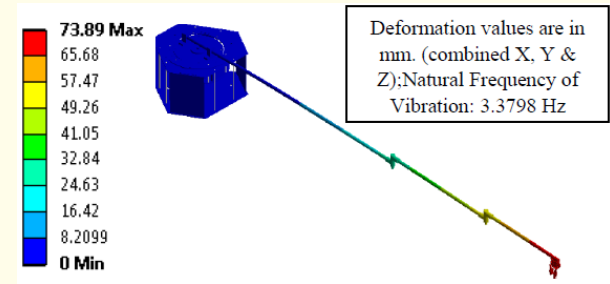

Figure 2: Deformation Pattern of the FRS at $1^{\text {st. }}$ Mode of Vibration.

Figures $3 \& 4$ present the deformation patterns of the prototype FRS, under $2^{\text {nd }} \& 3^{\text {rd }}$. modes of vibration respectively. 


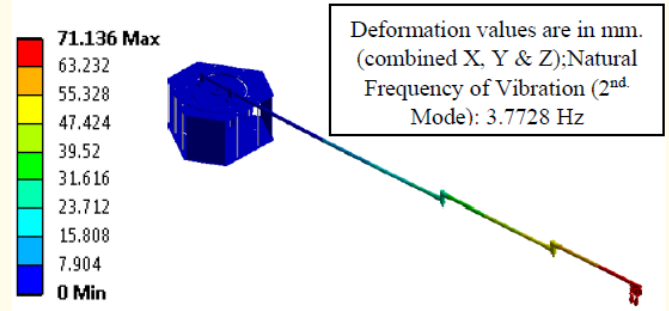

Figure 3: Deformation Pattern of the FRS at $2^{\text {nd }}$. Mode of Vibration.

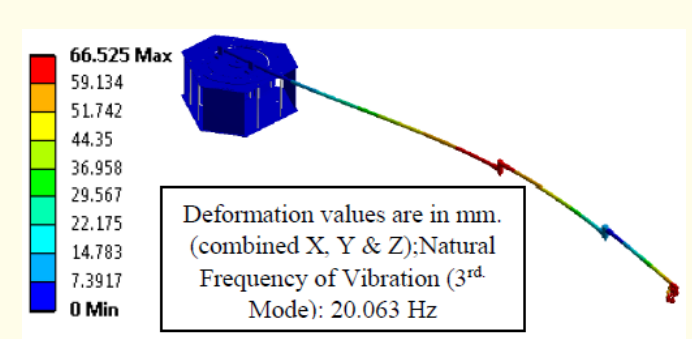

Figure 4: Deformation Pattern of the FRS at $3^{\text {rd }}$. Mode of Vibration.

The actual deformation in the total assembly can be obtained by FEA using frequency response technique. The modal analysis, done hitheto for our prototype, is pre-requisite of the frequency response technique. The analysis was carried out considering actual load of

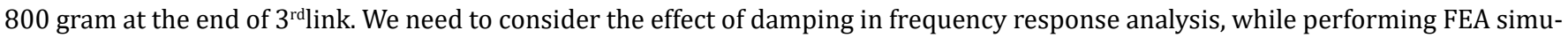
lation in real-time. $2 \%$ damping has been considered in the FEA. It is to be noted here that deflection of the system is apparent even at a lower frequency of vibration, e.g., $3 \mathrm{~Hz}$. Figure 5 illustrates the FEA-plot of such deflection at $3 \mathrm{~Hz}$. (Displacement values are in mm.) for the prototype FRS, without considering the system damping. Thus, the data of fig. 5can act as an upper bound for the ensemble deflection of the FRS. Nonetheless, it is important to accommodate damping in the system for an accurate simulation. We should also note that the predominant frequency of the system remained unaltered throughout the response analysis as there was no change in the phase angle.

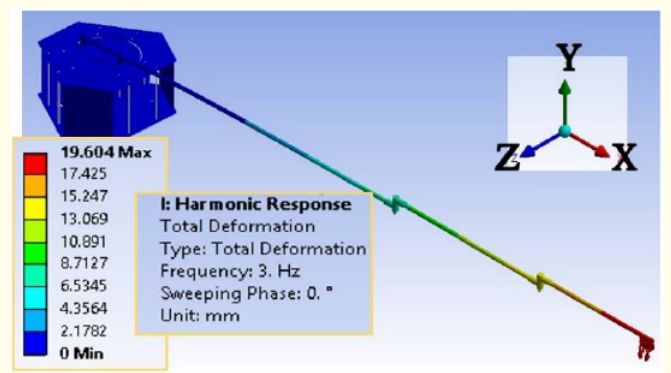

Figure 5: Upper Bound Deflection Values of the FRS at $3 \mathrm{~Hz}$.

The system has been found to be moderately stable in the range of higher frequencies, as can be seen from the graph of amplitude vs. frequency of fig. 6. Figure 7 shows the similar plot with $2 \%$ damping. Frequency (Hz.) \&Amplitude (mm.) values have been plotted along X \& Y-axes respectively for both the plots. Variation in amplitude of vibration has been studied under a frequency range of 1 to

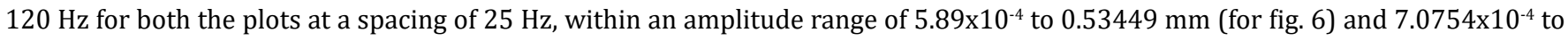
$0.50208 \mathrm{~mm}$. (for fig. 7). 


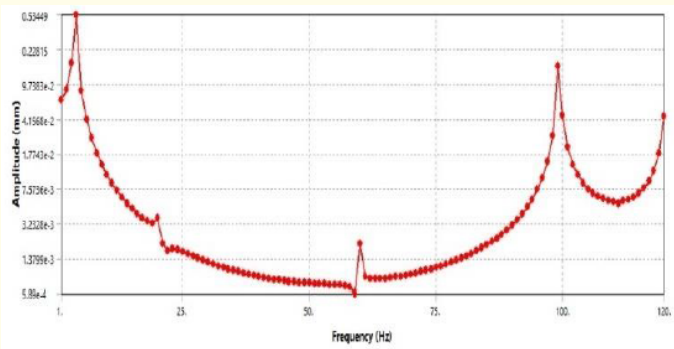

Figure 6: FEA-Results: Amplitude vs. Frequency Graph of the FRS.

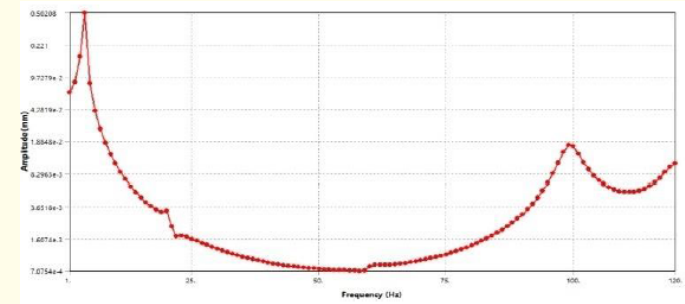

Figure 7: FEA-Results: Amplitude vs. Frequency Graph of the FRS at 2\% Damping.

\section{Design of the Device Controller \\ Proposition of Kinematic Model for the Design Syntax}

We have used homogeneous transformation for defining kinematic model of our prototype planar flexible manipulator using Denavit-Hartenberg (D-H) matrix. The said kinematic model was conceptualized as chain of semi-rigid bodies; namely, the semi-compliant links that are connected by revolute joints. The reference co-ordinate frame of the FRS-link(s) may revolve due to angular rotation and linear translation of the links, resulting in total spatial displacement of the end-effector. The systematic and generalized approach of D-H formulation helped us to find out an optimal measure for utilizing matrix algebra to describe the spatial geometry of the flexible links with respect to the fixed reference frame (located at the base of the prototype FRS). Accordingly, we have used a $4 \mathrm{x} 4$ homogeneous transformation matrix in order to describe the spatial relationship between two adjacent semi-rigid mechanical links that culminate in the desired forward kinematics of the prototype FRS. Figure 8 illustrates the methodology adopted for generating the kinematic model (forward as well as inverse) through a block diagram.

The evaluated D-H parameters will give the relative position and orientation of the links, considering Z-axis is in the direction of the joint axis and $\mathrm{X}$-axis is parallel to the common normal.

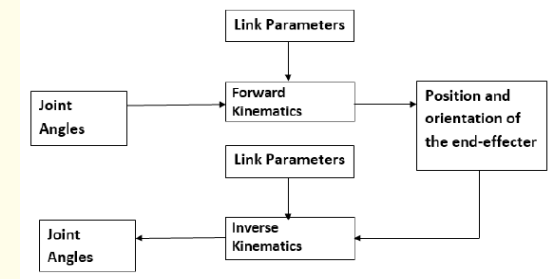

Figure 8: The Direct and Inverse Kinematics Syntax of the FRS. 
The D-H parameters to mounting configuration of link and determent based on the following rules

- $\quad q_{-} i$ is the angle from $x_{-}(i-1)$ to $x_{-} i$ along $z_{-}(i-1)$

- $\quad d_{-} i$ is distance from the intersection of $z_{-}(i-1)$ with $x_{-} i$ to the origin of (i-1) system of axes

- $\quad a_{-} i$ is the shortcut between $z_{-}(i-1)$ and $z_{-} i$

- $\quad \alpha_{-} \mathrm{i}$ is the angle from $z_{-}(\mathrm{i}-1)$ to $\mathrm{z}_{-} \mathrm{i}$ along $\mathrm{x}_{-} \mathrm{i}$.

Figure 9 shows the kinematic model scheme of the prototype 3 d.o.f flexible robot.

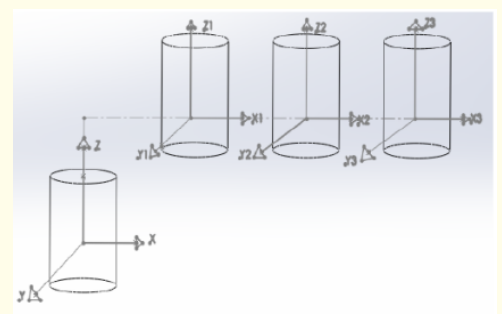

Figure 9: Kinematic Scheme of the Prototype Flexible Robot.

Accordingly, Table 1 presents the D-H parameters of the prototype FRS. With the help of said homogeneous transformation sequence, we get the final transformation matrix for the flexible robot.

\begin{tabular}{|c|c|c|c|c|}
\hline Joints & Joint angle & Offset & Link twist & Link length \\
\hline 1 & $\theta_{1}$ & 0 & 0 & $a_{1}$ \\
\hline 2 & $\theta_{2}$ & 0 & 0 & $a_{2}$ \\
\hline 3 & $\theta_{3}$ & 0 & 0 & $a_{3}$ \\
\hline
\end{tabular}

Table 1: Finalized D-H Parameters of the Prototype FRS.

\section{Developed Forward Kinematics}

Once the joint position, velocity, acceleration is known, we have computed the corresponding variables of the FRS-end-effecter in a given reference frame. By forward kinematic model, a function gets defined between the joint space $\mathrm{J}$ and work space $\mathrm{W}$, such that, $\mathrm{x}=$ $f(q)$ where $x € J, q € W$.

Figure 10 presents the simulation results of the developed kinematic model of the FRS at $0^{0}$ angle at all joints. Likewise, the simulation result on the developed kinematic model of the FRS at $90^{\circ}$ is shown in fig. 11.

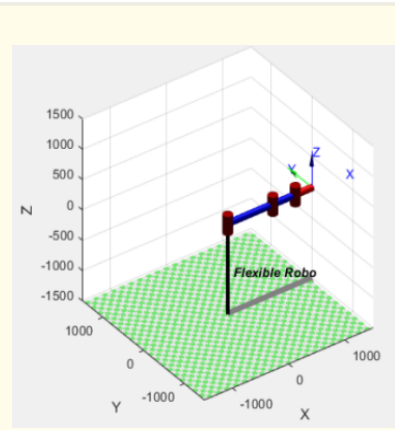

Figure 10: Simulation Results: Kinematic Model of the FRS at $0^{0}$ Angle for all Joints. 


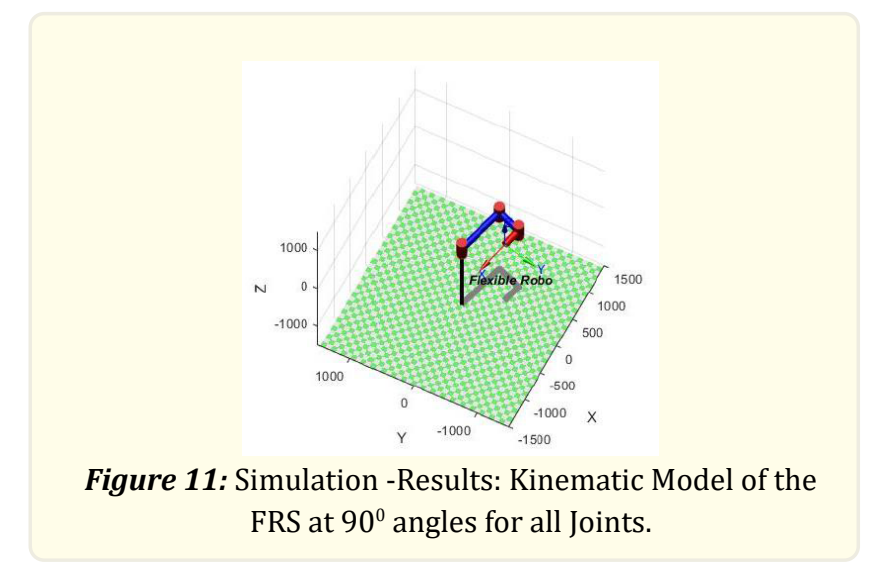

\section{Developed Inverse Kinematics}

Computation of the relevant variables (position, velocity, acceleration) pertaining to the prototype FRS, from the work space and the joint space was tackled by the indigenously developed inverse kinematic model, viz:

$$
\begin{gathered}
g=f-1 \text { from } J \text { to } W . \\
q=g(x)=f-1(x)
\end{gathered}
$$

Figure 12 illustrates the simulation results of the inverse kinematics model of the developed FRS.

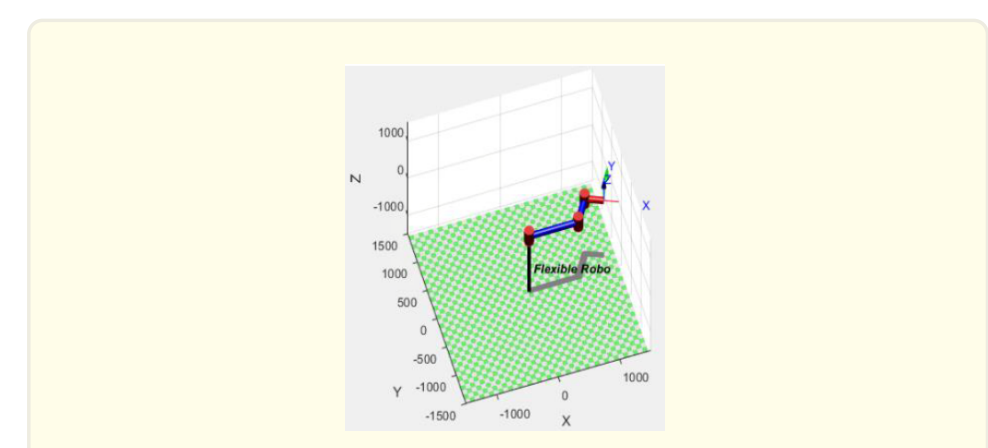

Figure 12: Simulation Results: Inverse Kinematic Model of the FRS.

\section{Input Shaping Design}

The methodology of 'Input Shaping' has been invoked for designing the customized control system architecture of the prototype FRS. This is essentially a feed forward control technique used for harnessing the residual vibration of flexible systems, in general. The generalized block diagram of input shaping control configuration is shown in fig. 13. Simulations on this basic control system layout of the prototype FRS were performed using MATLAB ${ }^{\circledR}$ simulation software. 


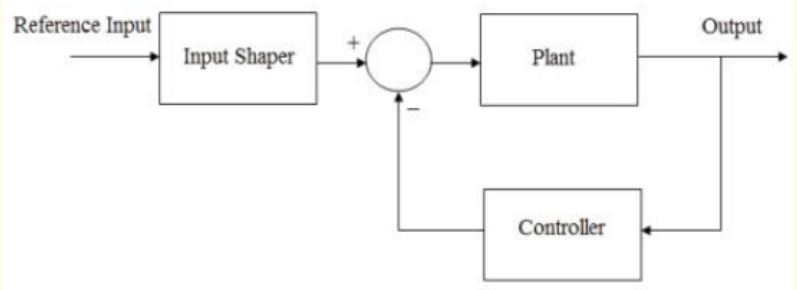

Figure 13: Block Diagram of a Generic Control System Using Input Shaping Technique.

Generally, a system with perpetual vibration of any order can be considered as a superposition of second order systems having transfer function, such as:

$$
G(s)=\frac{\omega^{2}}{s^{2}+2 \zeta \omega s+\omega^{2}}
$$

Where, $\omega$ is the natural frequency and $\zeta$ is the damping ratio of the vibrating system. The response of the system in time domain can be represented as,

$$
y(t)=\frac{A \omega}{\sqrt{1-\zeta^{2}}} \exp ^{-\zeta \omega\left(t-t_{0}\right)} \sin \left(\omega\left(t-t_{0}\right) \sqrt{1-\zeta^{2}}\right)
$$

Where, $A$ and $t_{0}$ are amplitude and time location of impulse respectively. As response of sequence is nothing but the superposition of the impulse responses, for ' $n$ ' number of responses, with damped frequency of vibration, $\omega_{d}=\omega\left(\sqrt{1-\zeta^{2}}\right)$, impulse response is given by,

$$
y(t)=M \sin \left(\omega_{d} t+\beta\right)
$$

Considering the series of impulse $(i=1,2, \ldots n)$, we get,

$$
\begin{aligned}
& M=\sqrt{\left\{\sum_{i=1}^{n} B_{i} \cos \varphi_{i}\right\}^{2}+\left\{\sum_{i=1}^{n} B_{i} \sin \varphi_{i}\right\}^{2}} \\
& B_{i}=\frac{\omega A_{i}}{\sqrt{1-\zeta^{2}}} \exp ^{-\zeta \omega\left(t-t_{0}\right)}, \varphi_{i}=\omega_{d} t_{i}
\end{aligned}
$$

Equtions $4 \mathrm{a} \& 4 \mathrm{~b}$ are instrumental in the evaluation of the frequency of vibration under the application of external forcing function, with specific importance on two parameters, namely, $A_{i}$ and $t_{i}$, which are the amplitudes and time locations of the impulses respectively. Now, in order to obtain zero vibration at the end of the terminal impulse in an ideal real-time situation, the necessary analytical requirement is related to the two terms of eqn. $4 \mathrm{a}$, viz. $\left\{\sum_{i=1}^{n} B_{i} \cos \varphi_{i}\right\}^{2}$ (say, V1) and $\left\{\sum_{i=1}^{n} B_{i} \sin \varphi_{i}\right\}^{2}$ (say, V2) that are independently be zero. In order to produce similar rigid body motion as unshaped reference command, sum of the amplitudes of the impulses is required to be unity, i.e.

$$
\sum_{i=1}^{n} A_{i}=1
$$

For improvement in the system response, the first impulse is selected at time $t_{1}(=0)$ and last impulse is at $t_{n}$.

The robustness of the FRS-controller can be improved by equating derivatives of V1 and V2 to zero. The robustness of the errors in natural frequencies of the system can be refined further by enhancing the order of the derivative thereof. After solving the derivatives for three consecutive sequences of impulse, we are able to find the following results: 


$$
\begin{aligned}
& \qquad t_{1}=0, t_{2}=\frac{\pi}{\omega_{d}}, t_{3}=\frac{2 \pi}{\omega_{d}} \\
& A_{1}=\frac{1}{1+2 K+K^{2}}, A_{2}=\frac{2 K}{1+2 K+K^{2}}, A_{3}=\frac{1}{1+2 K+K^{2}} \\
& \text { where } \mathrm{is} \text {, } \\
& K=e^{-\zeta \pi / \sqrt{1-\zeta^{2}}}
\end{aligned}
$$

\section{Simulation Results}

It may be stated that the numerical values of the natural frequencies of vibration of all the 10 modes were taken into consideration during the development of the control system of the prototype FRS. The impulse and step responses were obtained using MATLAB R2018a ${ }^{\circledR}$ simulation environment. Figures 14\& 15 show the impulse response of the prototype FRS controller before and after Input Shaping.

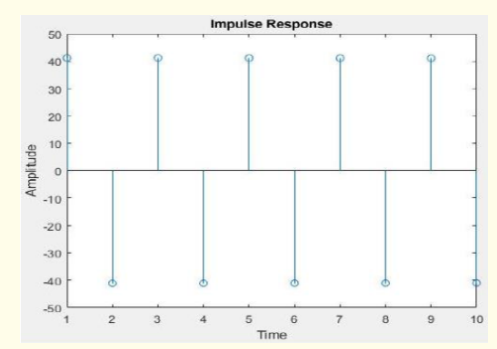

Figure 14: Impulse Response of the FRS before Input Shaping.

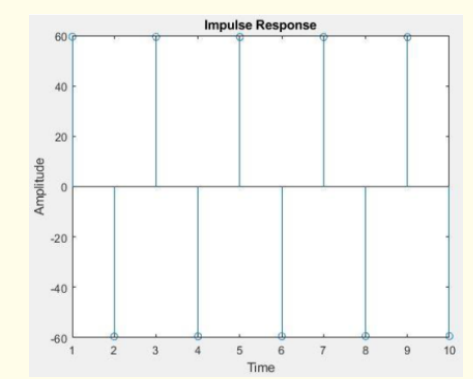

Figure 15: Impulse Response of the FRS after Input Shaping.

Figures 16 and 17illustrate step response of the prototype FRS controller before \& after Input Shaping. These plots render an idea of the vibrational amplitudes of the prototype FRS with respect to time.

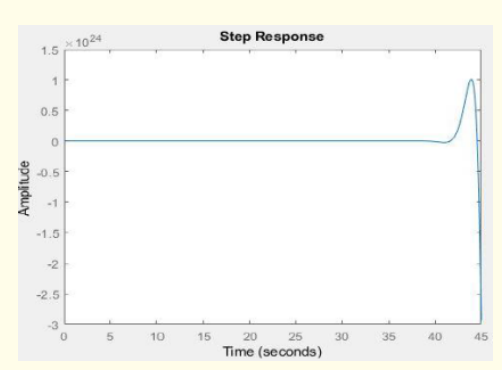

Figure 16: Step Response of the FRS before Input Shaping. 


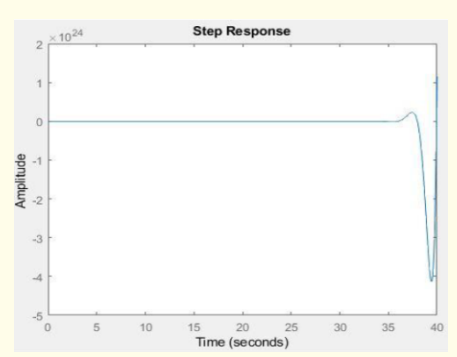

Figure 17: Step Response of the FRS after Input Shaping.

\section{Programming of the Device Controller}

\section{Program Semantics}

The indigenously designed \& developed controller of the prototype FRS has been orchestrated by the customized programming of the microcontroller. The design as well as computation for the FRS-control system are vital to use two programming environments in conjunction, namely, MATLABR2018 ${ }^{\circledR}$ and Arduino ${ }^{\mathrm{TM}}$ IDE. Accordingly, the programming for the prototype FRS was done in two segments: the first section dealt with complex mathematical computations in time and frequency domain for input shaping while the second part addressed the coding. The first section was programmed using MATLAB R2018 ${ }^{\circledR}$, which is a dynamic tool for computation. The results obtained from MATLAB ${ }^{\circledR}$ were used for passing parameters to the microcontroller by Arduino ${ }^{\mathrm{TM}}$ IDE. The output of MAT$\mathrm{LAB}^{\circledR}$ was used as variable in Arduino ${ }^{\mathrm{TM}}$ IDE. The system-generated feedback was obtained by the microcontroller and was sent back to $\mathrm{MATLAB}^{\circledR}$ for realization of the next loop. Figure 18 shows the flowchart of the program used for the controller of the prototype FRS.

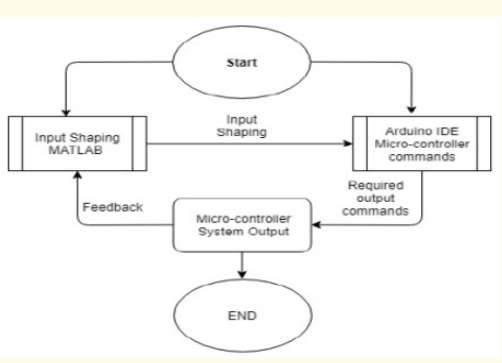

Figure 18: Flowchart for the Programming of the Prototype FRS.

Figure 19 shows the screenshot of the relevant program-code in MATLAB ${ }^{\circledR}$ for invoking Input Shaping. The representative sample of the microcontroller program, coded through Arduino IDE ${ }^{\mathrm{TM}}$, has been illustrated in fig. 20. 


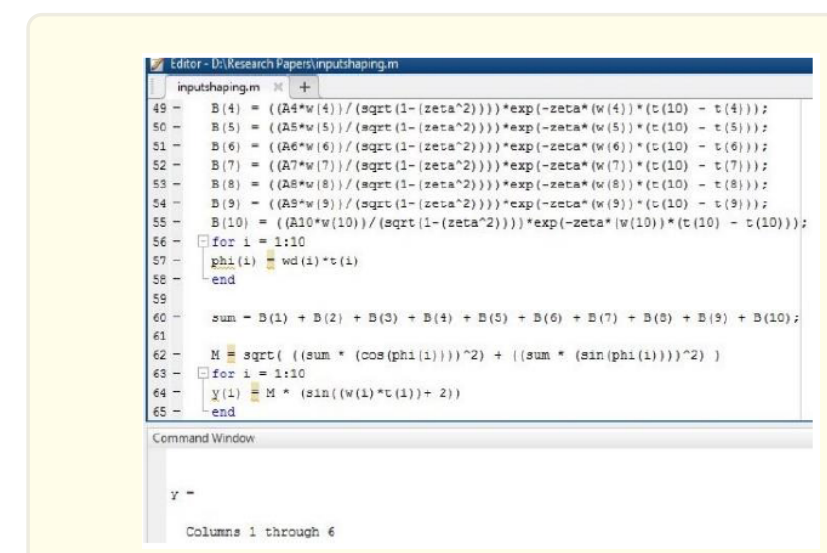

Figure 19: Screenshot of the MATLAB ${ }^{\circledR}$ Program Code for Input Shaping.

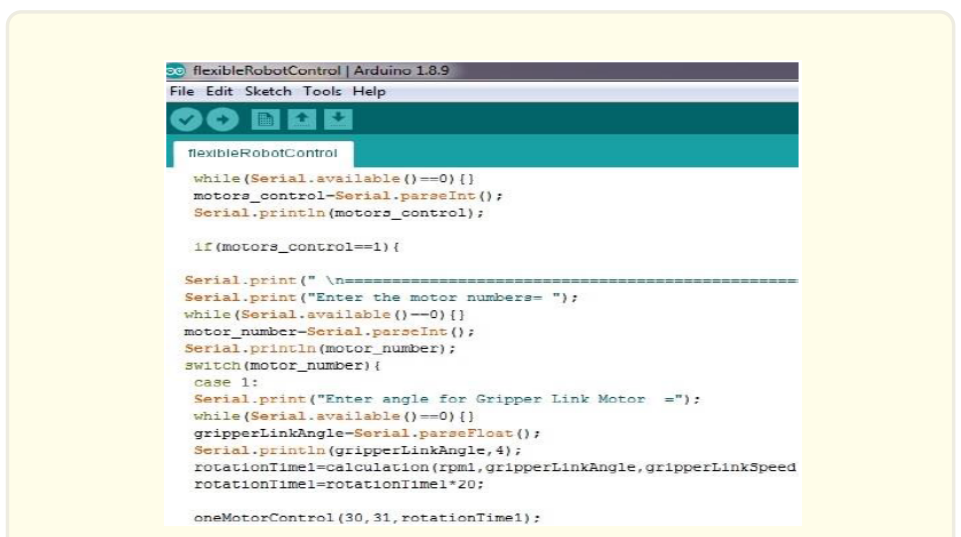

Figure 20: Screenshot of Microcontroller Program in Arduino ${ }^{\mathrm{TM}}$ IDE.

\section{Development of Graphical User Interface}

We have introduced the GUI application for controlling the prototype FRS in real-time. In order to keep programming tools minimal and to provide dynamic control over prototype FRS, GUI was composed using MATLAB R2018a ${ }^{\circledR}$. Figure 21 shows the block diagram of the GUI for our prototype FRS.

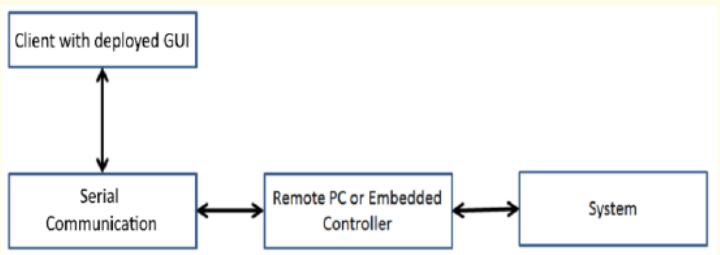

Figure 21: Block Diagram for GUI of the Prototype FRS.

The GUI consists of two sections for preforming the forward kinematics and inverse kinematics. Both sections are capable of accepting inputs from user, which are processed in MATLAB R2018 ${ }^{\circledR}$ that provides output in form of angles that can be passed to Arduino ${ }^{\mathrm{TM}}$ Idea's variables. Figure 22shows the screenshot of developed GUI for the prototype FRS. 


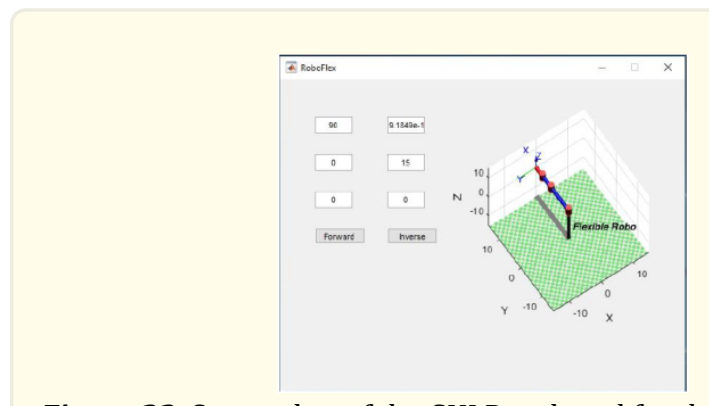

Figure 22: Screenshot of the GUI Developed for the Prototype FRS.

\section{Hardware Architecture of the Controller of the Prototype Planar Flexible Robot}

Indigenous design of the ensemble control circuit, instrumentation and interfacing of modules are the key facets of the controller hardware of the prototype FRS. The circuit design has been finalized taking into consideration of the functionality, operations, architecture and features of each device of the controller. Based on the syntax of the indigenous computer program, the hardware consists of serial communicator module and microcontroller module. The controller for the prototype FRS has been constructed using National Instruments ${ }^{\circledR} \mathrm{myRIO}^{\circledR}$, which provides dynamic interface with DC servomotors and motor drivers of the FRS. The myRIO ${ }^{\circledR}$ operates on Linux $^{\circledR}$-based operating system that gives way for specific operations such as multi-threading and multi-tasking. Besides, it has built-in serial communication module, which reduces the number of modules in the system.

The testing hardware of the prototype FRS is designed using ATMega2560 ${ }^{\mathrm{TM}}$ microcontroller and Arduino Mega ${ }^{\mathrm{TM}}$ built-in serial communication module. Arduino $\mathrm{Mega}^{\mathrm{TM}}$ is a programmable digital module, which incorporates the function of the central processing unit on a single Printed Circuit Board. ATMega2560 ${ }^{\mathrm{TM}}$ has 54 digital input-output (I/O) pins of which 15 can produce high speed pulses for pulse width modulation (PWM). ATMega2560 ${ }^{\mathrm{TM}}$ works with $\sim 16 \mathrm{MHz}$ clock speed that allows high speed data acquisition. Figure 23illustrates the block diagram of the hardware architecture of the controller of the prototype FRS.

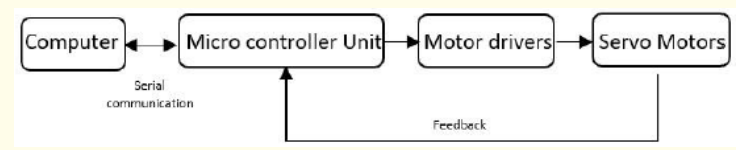

Figure 23: Block Diagram of the Hardware Architecture of Prototype FRS.

Actuators driving the links of the prototype FRS consist of DC servomotors. The motors were selected as per the torque and velocity requirement of the system. Notwithstanding the efficiency of the servomotors, the residual vibration in the system needs to be suppressed by the control system ensemble. Motor driver modules were selected optimally so as to provide a perfect interface between the motor and microcontroller unit. Power supply of the prototype FRS is a switched mode power supply with the voltage amplitude of $12 \mathrm{~V}$ DC and max current of $10 \mathrm{~A}$. Figure 24 shows the photographic view of the control system implemented on real- time prototype of the planar three-link FRS. 


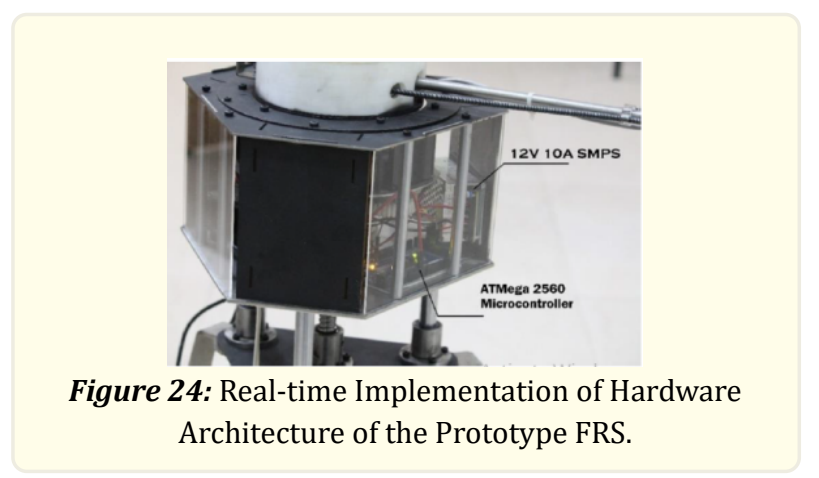

The photographic view of the ensemble hardware of the prototype planar three-link flexible robotic system is shown in fig. 25 .

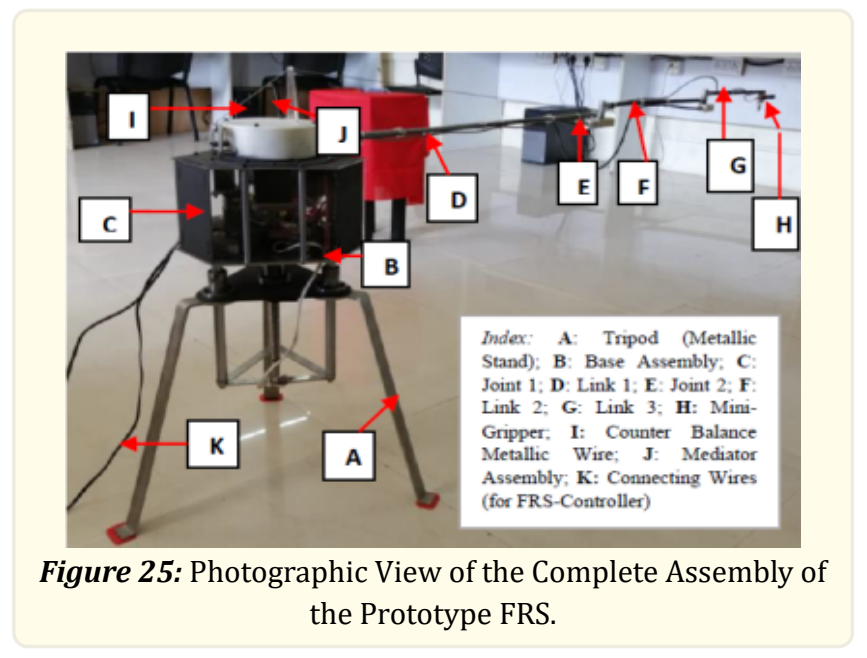

\section{Conclusions}

Design and prototype development of an indigenous robust control system for three-link serial flexible robotic system have been reported in this paper. The investigation of inherent vibration, its nature, natural frequencies and amplitudes were studied under various modes of vibration. A novel design of control system was established in real-time including input shaper that was implemented to dampen the natural vibrational frequencies obtained using FE-simulation. We have investigated the design-basis of the FRS-controller for kinematics and implemented the firmware in an elegant way. Developed control system provides accuracy as well as stability to the prototype FRS. The research-quotient earned through modeling \& hardware manifestation can be benchmarked. The software pathways as well as indigegous GUI have made the foundation for the firmware for the development of similar planar serial-chain flexible robots that can be deployed as assistive robot in near future.

\section{Acknowledgement}

We would like to express our heartfelt appreciation to Shri Shubham Chaudhari, Ex-CAE Support Engineer, SVR InfoTech, Pune, India for his valuable work on FE Analysis of the present research. Sincere appreciation is due to Shri Akshay Dalvi and Shri Amit Kumar Deka, Ex- Research Engineers, SVR Infotech for realizing some of the hardware issues of the developed controller and its software routines. We also thank Shri Pankaj Pandit, Shri Piyush Tailor, Shri Jaipal Solanki and Ms. Vibhavari Nirmale, Ex-Research Engineers, SVR Infotech for extending generous help in performing experiments with the FRS as well as run-time troubleshooting. 


\section{References}

1. NC Singer and WC Seering. "Preshaping Command Inputs to Reduce System Vibration". Journal of Dynamic Systems, Measurement \& Control- Transactions of the ASME, 112 (1990): 76-82.

2. Wen Chen. "Dynamic Modeling of Multi-link Flexible Robotic Manipulators". Computers \& Structures 79.2 (2001); 183-195.

3. Jianhua Zhang, Ying Tian and Minglu Zhang. "Dynamic Model and Simulation of Flexible Manipulator based on Spring \& Rigid Bodies". Proceedings of the 2014 IEEE International Conference on Robotics \& Biomimetics ('ROBIO-2014') (2014): 2460-2464.

4. H Tjahyadi and K Sammut. "Multi-mode Vibration Control of a Flexible Cantilever Beam using Adaptive Resonant Control". Smart Materials and Structures 15 (2006): 270-278.

5. Juan R Trapero-Arenas, MamadouMboup, Emiliano Pereira-Gonalez and Vicente Feliu. "Online Frequency and Damping Estimation in a Single-Link Flexible Manipulator based on Algebraic Identification". Proceedings of the 16th. Mediterranean Conference on Control \& Automation (IEEE), Franco (2008): 338-343.

6. Emiliano Pereirea, Summet Sunil Aphale, Vicente Feliu and SOR Moheimani. "Integral Resonant Control for Vibration Damping and Precise Tip-positioning of a Single-link Flexible Manipulator". IEEE/ ASME Transactions on Mechatronics 16.2 (2011): 232 240.

7. Smith OJM. Feedback Control Systems, McGraw-Hill, New York (1958): 338.

8. AR Fraser and RW Daniel. "Perturbation Techniques for Flexible Manipulators". Norwell, MA, Kluwer (1991).

9. Junkins John L and Turner James D. Optimal Spacecraft Rotational Maneuvers, Elsevier Science Publishers, New York (1986).

10. Chun Hon M, Turner, James D and Juang Jer-Nan. "Disturbance Accommodating Tracking Maneuvers of Flexible Spacecraft". Journal of the Astronautical Sciences 33.2 (1985): 197-216

11. Gupta, Narendra K. "Frequency-Shaped Cost Functionals: Extension of Linear-Quadratic-Gaussian Design Methods". Journal of Guidance and Control 3.6 (1980): 529-335.

12. Juang Jer-Nan, Turner, James D and Chun, Hon M. "Closed-FormSolutions for Feedback Control with Terminal Constraints". Journal of Guidance and Control 8.1 (1985): 39-43.

13. VG Moudgal, WA Kwong, KM Passino and S Yurkovich. "Fuzzy Learning Control for a Flexible-link Robot". IEEE Transactions on Fuzzy Systems 3.2 (1995): 199-210.

14. M Benosman and G Vey. "Control of Flexible Manipulators: A Survey". Robotica 22 (2004): 533-545.

15. PT Kotnik, S Yurkovich and U Ozfuner. "Acceleration Feedback for Control of a Flexible Manipulator Arm". Journal of Robotic Systems 5.3 (1988):181-196.

16. JJ Feliu, V Feliu and C Cerrada. "Load Adaptive Control of Single-Link Flexible Arms Based on a New Modeling Technique". IEEE Transactions on Robotics and Automation 15 (1999): 793-804.

17. L-C Lin and T-W Yih. "Rigid Model-based Neural Network Control of Flexible-link Manipulators", IEEE Transactions of Robotics and Automation 12 (1996): 595-602.

18. MM Moallem, R Patel and K Khorasani. "An Inverse Dynamics Control Strategy for Tip Position Tracking of Flexible Multi-link Manipulators". Journal of Robotic Systems 14.9 (1997):pp. 649-658.

19. W Yim and SN Singh. "Predictive End-point Trajectory Control of Elastic Manipulators". Journal of Robotic Systems 13.9 (1996): 561-569.

20. Vicente Feliu, José A Somolinos and Andrés García. "Inverse Dynamics Based Control System for a Three-Degree-of-Freedom Flexible Arm". IEEE Transactions on Robotics and Automation 19.6 (2003): 1007-1014.

21. J Somolinos, V Feliu and L Sánchez. “Design, Dynamic Modeling and Experimental Validation of a New Three-Degree-of-Freedom Flexible Arm". Mechatronics 12.7 2002): 919-948.

22. Warude P, Patel M, Pandit P, Patil V, Pawar H, Nate C, Gajlekar S, Atpadkar V and Roy D. "On the Design and Vibration Analysis of a Three-Link Flexible Robot Interfaced with a Mini-Gripper". Proceedings of the 8th. National Conference on Wave Mechanics \& Vibration (“WMVC-2018”), NIT-Rourkela, Odisha, India (2018): 26-28.

23. Rauniyar A, Roy D, Pandit P and Atpadkar V. “Design Model for the Drive \& Actualtor of the Test Set-up of a Novel Flexible Ro- 
botic System". Proceedings of the 2018 IEEE International Conference on Computational Intelligence and Computing Research (IEEE-ICCIC-2018), Madurai, Tamil Nadu, India (2018): 13-15.

24. Roy Debanik. "Towards the Control of Inherent Vibration of Flexible Robotic Systems and Associated Dynamics: New Proposition and Model". International Journal of Robotics Research, Applications and Automation 1.1 (2019): 6-17.

Volume 2 Issue 2 February 2022

(C) All rights are reserved by Moiseev Pratik Chothe., et al. 